\title{
Control of Differentiation of Human Mesenchymal Stem Cells by Altering the Geometry of Nanofibers
}

\author{
Satoshi Fujita, ${ }^{1}$ Harue Shimizu, ${ }^{1}$ and Shin-ichiro Suye ${ }^{2}$ \\ ${ }^{1}$ Department of Fibre Amenity Engineering, Graduate School of Engineering, University of Fukui, Fukui 910-8507, Japan \\ ${ }^{2}$ Department of Applied Chemistry and Biotechnology, Graduate School of Engineering, University of Fukui, Fukui 910-8507, Japan
}

Correspondence should be addressed to Satoshi Fujita, fujitas@u-fukui.ac.jp

Received 30 April 2012; Accepted 21 May 2012

Academic Editor: A. M. Rao

Copyright (C) 2012 Satoshi Fujita et al. This is an open access article distributed under the Creative Commons Attribution License, which permits unrestricted use, distribution, and reproduction in any medium, provided the original work is properly cited.

\begin{abstract}
Effective differentiation of mesenchymal stem cells (MSCs) is required for clinical applications. To control MSC differentiation, induction media containing different types of soluble factors have been used to date; however, it remains challenging to obtain a uniformly differentiated population of an appropriate quality for clinical application by this approach. We attempted to develop nanofiber scaffolds for effective MSC differentiation by mimicking anisotropy of the extracellular matrix structure, to assess whether differentiation of these cells can be controlled by using geometrically different scaffolds. We evaluated MSC differentiation on aligned and random nanofibers, fabricated by electrospinning. We found that induction of MSCs into adipocytes was markedly more inhibited on random nanofibers than on aligned nanofibers. In addition, adipoinduction on aligned nanofibers was also inhibited in the presence of mixed adipoinduction and osteoinduction medium, although osteoinduction was not affected by a change in scaffold geometry. Thus, we have achieved localized control over the direction of differentiation through changes in the alignment of the scaffold even in the presence of a mixed medium. These findings indicate that precise control of MSC differentiation can be attained by using scaffolds with different geometry, rather than by the conventional use of soluble factors in the medium.
\end{abstract}

\section{Introduction}

Mesenchymal stem cells (MSCs) are multipotent, allowing differentiation into various types of cells, such as adipocytes, osteoblasts, and chondrocytes [1]. This ability is expected to be useful in many clinical applications, including the regeneration of damaged and injured tissues, and in cosmetic surgery [2-6]. At present, MSCs are directly administrated into affected tissues, but cells differentiated from MSCs have not yet been attempted in clinical applications, because of the difficulties posed by the precise control of induction. However, if such differentiated cells were available for clinical applications, the time to engraftment of cells onto the affected tissues could be reduced. To date, to control MSC differentiation, an induction medium, containing several types of soluble factors, has been employed and has efficiently induced differentiation [1]. However, obtaining a uniformly differentiated population of cells, of a quality appropriate for clinical applications, by using only soluble factors, has been challenging. In addition, residual soluble factors also pose safety issues [7].

Recently, McBeath et al. reported that MSCs attached to a wide flat surface differentiated into osteoblasts, while cells attached to a small surface differentiated into adipocytes [8]. They also reported that cell shape is a key factor in cell differentiation, because the intracellular tension triggers changes in expression of GTPases, such as RhoA and its downstream Rho kinases, which results in changes in cellular behaviors.

This knowledge can also be applied to the 3-dimensional (3D) culture of MSCs, not only to cultures grown on a flat surface. However, cells cultured in a dish show quite different morphologies to cells grown in a 3D culture. Cells in a living tissue are surrounded by an extracellular matrix (ECM), which has a fibrous network structure; such cells take on elongated shapes in a particular direction, in accordance with the fibrous structure of the ECM. In order to provide a scaffold material for use in 3D culture, hydrogels have typically been prepared from synthetic and natural polymers 
$[9,10]$. Although hydrogels are easy to prepare and handle, they are structurally uniform, and not anisotropic. For this reason, cells do not keep elongating in hydrogels, as they do in living tissues.

In this study, in order to address this problem, and to control cell differentiation in a $3 \mathrm{D}$ environment using biomaterials that mimic living tissues, we have attempted to develop a scaffold for effective differentiation, using synthetic nanofibers. We prepared nanofiber scaffolds with a high level of anisotropy, which mimics the structure of the ECM and nanometer-sized elastic fibers, such as collagen, and compared MSC differentiation on these media. We used an electrospinning method to prepare these nanofiber scaffolds, because this approach allows easy control of the diameter, density, and geometric structure of the fiber [11-13]. We also investigated the effect of scaffold geometry, and the alignment of nanofibers, on cell differentiation. We hypothesized that cells elongated along with aligned nanofibers receive higher differentiation signal via cytoskeletal tension than cells on random nanofibers.

\section{Experimental}

2.1. Fabrication of Nanofiber Scaffold. Polyurethane (PU; P22SRNAT, JIS hardness 82A; Nippon Polyurethane Industry, Tokyo, Japan) was purified by methanol re-precipitation from a tetrahydrofuran solution (THF; Wako Pure Chemical Industries, Osaka, Japan), and dried under reduced pressure prior to use. PU was dissolved in 95\% THF and 5\% N,Ndimethylformamide (DMF; Wako) at 12.5\% (w/v). PU nanofibers were electrospun onto a glass coverslip (No. $5 ; 22 \times$ $26 \times 0.45-0.60 \mathrm{~mm}$; Matsunami, Osaka, Japan) using an electrospinning setup (MECC, Fukuoka, Japan) as detailed in Scheme 1. The electrospinning unit consisted of a highvoltage power supply, a rotating collector, a syringe pump, and a $23 \mathrm{G}$-needle that was connected to a syringe by a tube. A high voltage, $25 \mathrm{kV}$, was applied between the syringe needle and the ground electrode. A rotating collector (diameter: $10 \mathrm{~cm}$ ) was placed horizontally, $10 \mathrm{~cm}$ from the needle. The $\mathrm{PU}$ solution was extruded from the syringe at a rate of $0.6 \mathrm{~mL} / \mathrm{h}$. The speed and duration of rotation were $1,500 \mathrm{rpm}$ and $3 \mathrm{~min}$, respectively, for the aligned nanofiber scaffold, and $0 \mathrm{rpm}$ (static) and $30 \mathrm{~s}$, respectively, for the random nanofiber scaffold. To improve the surface hydrophilicity of the fibers, oxygen plasma treatment $(100 \mathrm{~W}, 30 \mathrm{~s}, 5 \mathrm{~Pa}$, chamber size: $\varphi 64 \mathrm{~mm} \times 160 \mathrm{~mm}$ ) was carried out using a plasma reactor (PR300; Yamato Scientific, Tokyo, Japan).

2.2. Scanning Electron Microscopy. The fabricated nanofiber scaffold was sputter-coated with $\mathrm{Pt} / \mathrm{Pd}$ using an ion sputter (E-1030; Hitachi, Tokyo, Japan). Scanning electron microscopy (SEM) observation was carried out with an S-2600HS microscope (Hitachi).

2.3. Measurement of Fiber Diameter and Orientation. Fiber diameters and angles were measured from SEM images using

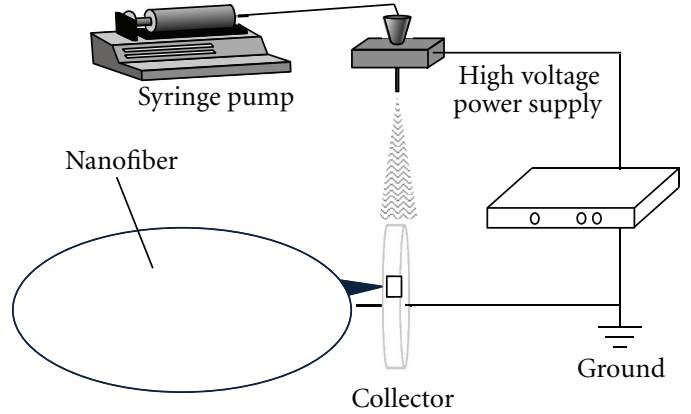

Scheme 1: Setup of electrospinning.

ImageJ software (ver. 1.46a). Fiber orientation was quantified using the second-order parameter $S$ as follows:

$$
S=\left(3\left\langle\cos ^{2} \theta\right\rangle-1\right) / 2
$$

where $\theta$ is an angle of each fiber and $\left\langle\cos ^{2} \theta\right\rangle$ is the average of $\cos ^{2} \theta$.

2.4. Cell Culture. Human bone-marrow-derived MSCs (Takara Bio, Shiga, Japan) were maintained in Dulbecco's Modified Essential Medium (DMEM; Invitrogen, CA, USA) supplemented with $10 \%$ fetal bovine serum (FBS; BIOWEST, France), $100 \mathrm{U} / \mathrm{mL}$ penicillin, and $100 \mu \mathrm{g} / \mathrm{mL}$ streptomycin (Invitrogen) at $37^{\circ} \mathrm{C}$ under $5 \% \mathrm{CO}_{2}$, in a humidified atmosphere, and subcultured at $3 \times 10^{3}$ cells per $\mathrm{cm}^{2}$ every 3-4 days. Cells at passages $2-7$ were used for experiments [18].

2.5. Differentiation on Nanofiber Scaffolds. An oxygen plasmatreated nanofiber scaffold, placed in a $35 \mathrm{~mm} \varphi$ dish was washed with $70 \%$ ethanol, twice with phosphate buffered saline (PBS), and then with DMEM/10\% FBS. Cells were seeded onto this scaffold at $2 \times 10^{4}$ cells per $\mathrm{cm}^{2}$ and cultured at $37^{\circ} \mathrm{C}$ for $6 \mathrm{~h}$. Then, the medium was exchanged for differentiation medium. For osteogenic differentiation, osteoinduction medium (OIM; DMEM supplemented with $100 \mathrm{nM}$ dexamethasone [Sigma-Aldrich, MO, USA], $50 \mu \mathrm{M}$ ascorbic acid 2-phosphate [Wako], $10 \mathrm{mM} \beta$-glycerophosphate [Wako] and 10\% FBS) was used. For adipogenic differentiation, adipoinduction medium (AIM; DMEM containing $1 \mu \mathrm{M}$ dexamethasone [Sigma-Aldrich], $0.2 \mathrm{mM}$ indomethacin [Wako], $10 \mu \mathrm{g} / \mathrm{mL}$ insulin [Sigma-Aldrich], 0.5 mM 3-isobutyl-1-methylxanthine [Sigma-Aldrich], and $10 \%$ FBS) was used. Culture media were changed freshly every 2 or 3 days [15].

2.6. Histochemical Analysis. The presence of mineralized deposits after osteogenic differentiation was determined with von Kossa staining [15]. In a brief, cultures were rinsed twice with PBS, then fixed with 10\% formaldehyde for $30 \mathrm{~min}$ and thereafter washed 3 times with distilled water. One milliliter of $5 \%(\mathrm{w} / \mathrm{v})$ silver nitrate (Nacalai Tesque, Kyoto, Japan) was added to each well. The cultures were placed in the dark for $30 \mathrm{~min}$, washed with water, and then developed with $5 \%$ $(\mathrm{w} / \mathrm{v})$ sodium carbonate in $25 \%$ formaldehyde for $5 \mathrm{~min}$. 
Table 1: Primer list.

\begin{tabular}{|c|c|c|c|c|c|c|}
\hline Gene & Primer sequences $\left(5^{\prime}-3^{\prime}\right)$ & HGNC ID & Annealing temp $\left({ }^{\circ} \mathrm{C}\right)$ & Cycle number & Product size $(\mathrm{bp})$ & Reference \\
\hline ALPL & $\begin{array}{c}\text { ATCTTTGGTCTGGCCCCCATG } \\
\text { ATGCAGGCTGCATACGCCAT }\end{array}$ & HGNC: 438 & 59 & 35 & 287 & {$[14]$} \\
\hline RUNX2 & $\begin{array}{l}\text { CAGTTCCCAAGCATTTCATCC } \\
\text { TCAATATGGTCGCCAAACAG }\end{array}$ & HGNC: 10472 & 55 & 35 & 444 & {$[15]$} \\
\hline SPP1 & $\begin{array}{l}\text { AAGCGAGGAGTTGAATGG } \\
\text { GGAAAGTTCCTGACTATC }\end{array}$ & HGNC: 11255 & 51 & 35 & 220 & {$[14]$} \\
\hline BGLAP & $\begin{array}{l}\text { GCCGTAGAAGCGCCGATAGGC } \\
\text { ATGAGAGCCCTCACACTCCTC }\end{array}$ & HGNC: 1043 & 49 & 35 & 294 & {$[14]$} \\
\hline COL1A1 & $\begin{array}{l}\text { CCACCAATCACCTGCGTACAGAAC } \\
\text { GGCACGGAAATTCCTCCGGTTGAT }\end{array}$ & HGNC: 2197 & 63 & 35 & 428 & {$[14]$} \\
\hline COL3A1 & $\begin{array}{l}\text { CTGGACCAAAAGGTGATGCT } \\
\text { CAGGGTTCCATCTCTTCCA }\end{array}$ & HGNC: 2201 & 56 & 35 & 291 & {$[14]$} \\
\hline FABP4 & $\begin{array}{l}\text { ACCAGGAAAGTGGCTGGCAT } \\
\text { CAGGTCAACGTCCCTTGGCT }\end{array}$ & HGNC: 3559 & 58 & 35 & 331 & {$[16]$} \\
\hline CEBPA & $\begin{array}{l}\text { CGGTGGACAAGAACAGCAAC } \\
\text { CGGAATCTCCTAGTCCTGGC }\end{array}$ & HGNC: 1833 & 58 & 35 & 365 & {$[16]$} \\
\hline PPARG & $\begin{array}{l}\text { GCTGTTATGGGTGAAACTCTG } \\
\text { ATAAGGTGGAGATGCAGGTTC }\end{array}$ & HGNC: 9236 & 55 & 35 & 351 & {$[15]$} \\
\hline ADIPOQ & $\begin{array}{l}\text { AGCCTCCTTCTCCTGGGTCC } \\
\text { GTTGCCTCTAGCCTGGTGGG }\end{array}$ & HGNC: 13633 & 58 & 35 & 295 & {$[16]$} \\
\hline LPL & $\begin{array}{l}\text { GAGATTTCTCTGTATGGCACC } \\
\text { CTGCAAATGAGACACTTTCTC }\end{array}$ & HGNC: 6677 & 50 & 35 & 276 & {$[16]$} \\
\hline GAPDH & $\begin{array}{l}\text { AGCCGCATCTTCTTTTGCGTC } \\
\text { TCATATTTGGCAGGTTTTTCT }\end{array}$ & HGNC: 4141 & 55 & 28 & 816 & {$[15]$} \\
\hline
\end{tabular}

After development, cultures were washed, 5\% sodium thiosulfate added to stop development, again washed with water, and then air-dried and imaged. The amount of calcium deposits was evaluated from image analyses using ImageJ software. For the detection of alkaline phosphatase activity, AP-Substrate Kit III (SK-5300, Vector laboratories, CA, USA) was used following the manufacturer's instructions. For the detection of adipogenic induction, cells were fixed in $4 \%$ paraformaldehyde and incubated with Oil-Red-O (Wako) to stain lipid vacuoles [15].

2.7. RT-PCR. Gene expression in MSCs was analyzed by reverse transcriptase polymerase chain reaction (RT-PCR). Total RNA was extracted from MSCs using the High Pure RNA Isolation Kit (Roche Diagnostics, Tokyo, Japan), which includes DNase activity, according to the manufacturer's instructions. Complementary DNA (cDNA) was then synthesized from the extracted total RNA using the Transcriptor Universal cDNA Master kit (Roche). Specific cDNA was amplified by PCR using a reaction mix $(20 \mu \mathrm{L})$ composed of $2 \mathrm{mM}$ Tris- $\mathrm{HCl}$ ( $\mathrm{pH} 8.0$ ), $10 \mathrm{mM} \mathrm{KCl,} 0.01 \mathrm{mM}$ EDTA, $0.1 \mathrm{mM}$ DTT, $1 \mathrm{U}$ DNA polymerase (TaKaRaEx Taq; Takara Bio), $0.2 \mathrm{mM}$ dNTP mixture, $0.5 \mu \mathrm{M}$ of each primer, and approximately $100 \mathrm{ng}$ of cDNA template. The temperature settings for each cycle were $94^{\circ} \mathrm{C}$ for $45 \mathrm{~s}$ for the denaturing step, $49-63^{\circ} \mathrm{C}$ for $30 \mathrm{~s}$ for the annealing step, and $72^{\circ} \mathrm{C}$ for 90 $s$ for the extension step. All primer sequences and their PCR conditions are listed in Table 1 [14-16]. Amplified products $(9 \mu \mathrm{L})$ were electrophoresed in $2 \%$ SeaKem GTG agarose gels containing $0.5 \mu \mathrm{g} / \mathrm{mL}$ ethidium bromide, at $100 \mathrm{~V}$ for $30 \mathrm{~min}$, and the PCR products visualized on an ultraviolet illuminator. The optimal PCR cycle conditions were adjusted according to the brightness of the visualized band.

2.8. DNA Microarray. Gene expression in MSCs cultured in the maintaining medium for 3 days on aligned and random nanofiber scaffolds were compared by DNA microarray. In brief, the total RNA extracted from MSCs as described above was quantified using an Agilent 2100 Bioanalyzer (Agilent Technologies). cDNA was generated from $100 \mathrm{ng}$ of the total RNA using Ambion's Whole Transcripts Expression Kit (Ambion); these were fragmented and end-labeled using a GeneChip WT Terminal Labeling Kit (Affymetrix), according to the manufacturer's instructions. An aliquot of the labeled DNA $(5.5 \mu \mathrm{g})$ was hybridized to an Affymetrix GeneChip Human Gene 1.0 ST array (genome-wide expression profiling chip, 28,869 genes with 764,885 probes; Affymetrix) at $45^{\circ} \mathrm{C}$ for $16 \mathrm{~h}$. After hybridization, the array was stained with Fluidics 450 station and scanned using a GCS3000 Scanner (Affymetrix). Scanned CEL files were processed with Affymetrix Expression Console software to obtain RMA signals. Differentially expressed genes with a fold-change greater than \pm 1.5 were identified with a Subio Platform (Subio Inc., Tokyo, Japan). 


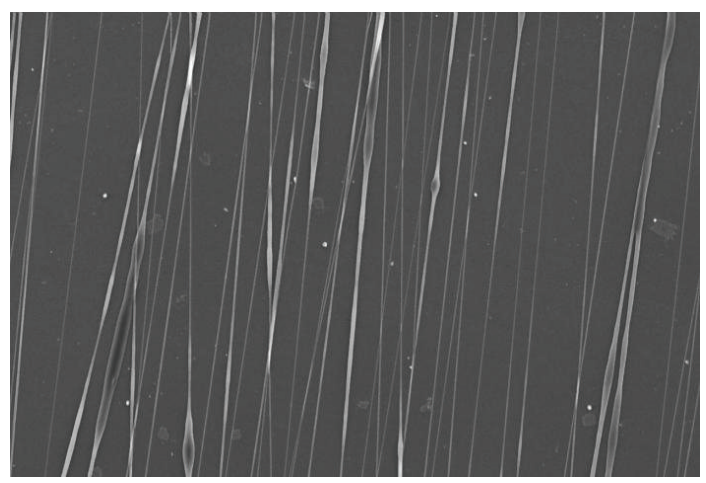

(a)

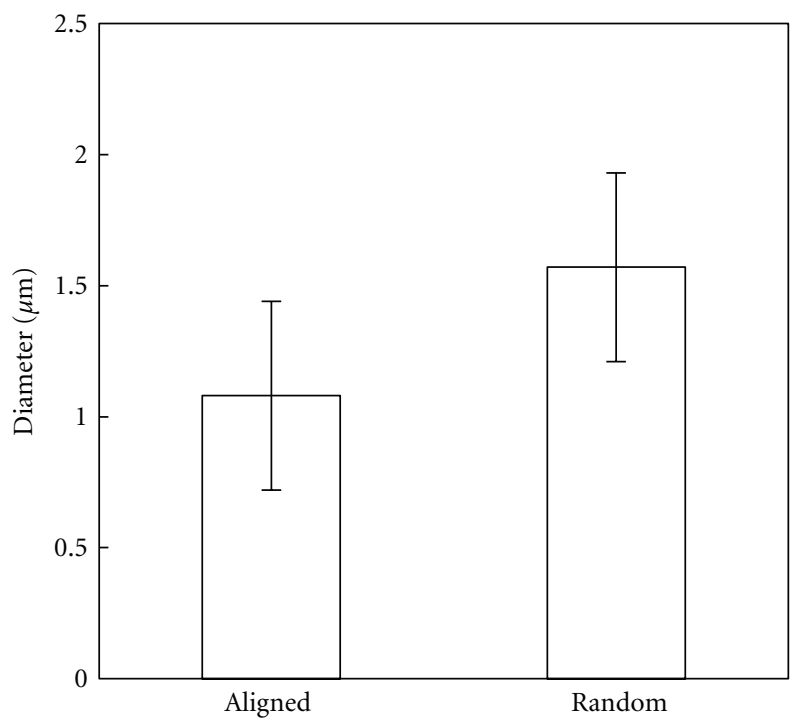

(c)

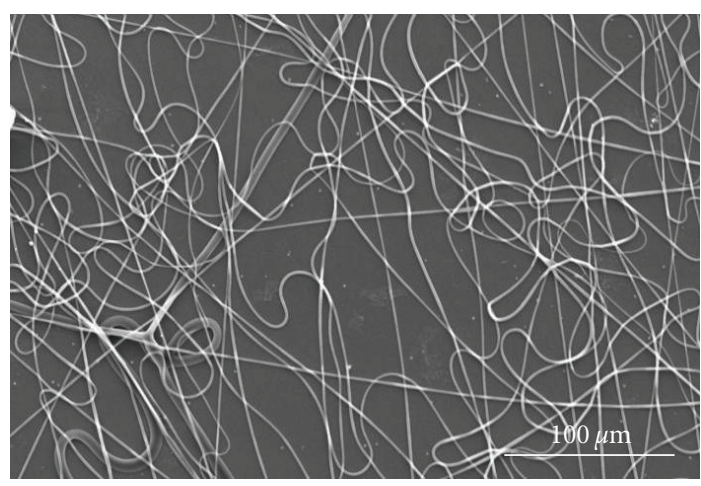

(b)

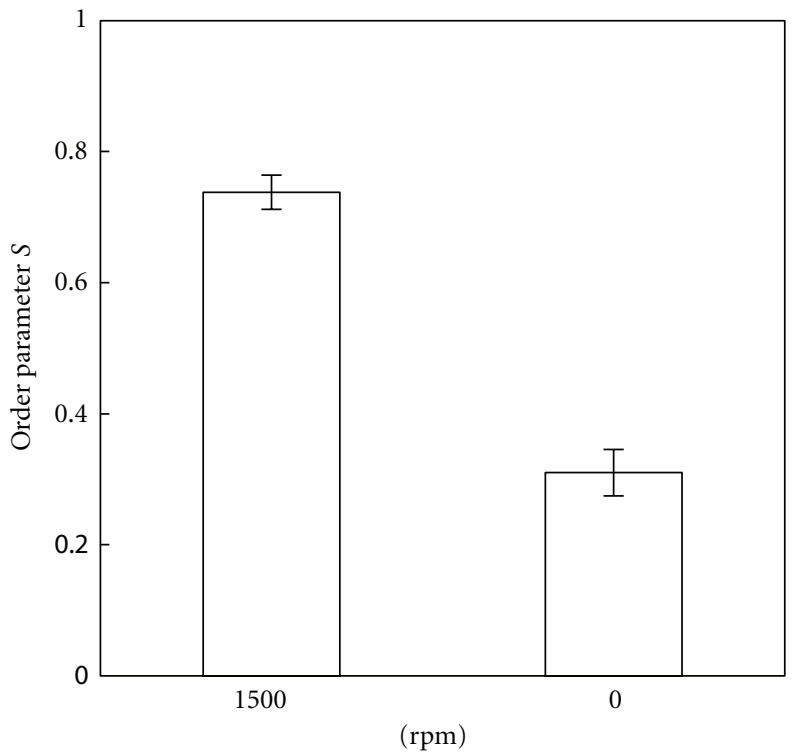

(d)

FIGURE 1: ((a), (b)) SEM images of nanofibers fabricated on (a) a rotating collector (1,500 rpm) and (b) a static collector. (c) Fiber diameter. Means \pm standard deviations $(\mathrm{SD})(n=10)$. (d) Order parameter of fiber scaffolds.

\section{Results and Discussion}

3.1. Fabrication of Nanofiber Scaffolds. Nanofiber scaffolds for cell culture were fabricated by electrospinning from $12.5 \% \mathrm{PU}$ solution using either a rotating or a static collector (Scheme 1). The diameters of fibers were measured from the SEM images, such as those shown in Figures 1(a) and 1(b). There was no difference in the diameters of nanofibers collected on a rotating or on a static collector (mean 1.08 \pm SD $0.36 \mu \mathrm{m}$ and $1.57 \pm 0.36 \mu \mathrm{m}$, resp., Figure $1(\mathrm{c}))$. The orientation of the fibers was quantified by calculating a secondorder parameter, $S$, from the fiber angles that were measured in the SEM images; a higher value (approaching 1) of $S$ implies a higher orientation along a specific direction, while a lower value (approaching 0 ) implies random orientation. As shown in Figure 1(d), fibers fabricated on a rotating collector demonstrated higher alignment $(S=0.74 \pm 0.03)$ than did fibers fabricated on a static collector $(S=0.31 \pm 0.04)$. Therefore, fibers fabricated on a rotating collector and on a static collector were designated as "aligned" and "random" fibers, respectively.

3.2. Cell Differentiation on a Nanofiber Scaffold. To examine the effect of scaffold geometry on MSC differentiation, MSCs were seeded on the aligned and random nanofiber scaffolds and induced to differentiate into osteoblasts or adipocytes by using appropriate differentiation medium containing specific soluble factors. To minimize the local fluctuation of soluble factors in the culture medium, in order to evaluate only the effect of the scaffold geometry, 2 types of nanofiber scaffolds were placed on the same dish and cells on each scaffold were cultured in the way, sharing the same medium. After a 14-day osteoinduction with OIM, von Kossa staining was performed to evaluate the deposition of calcium. Some black spots of calcium deposition were observed, as shown in Figure 2(A), but there was no significant difference in 


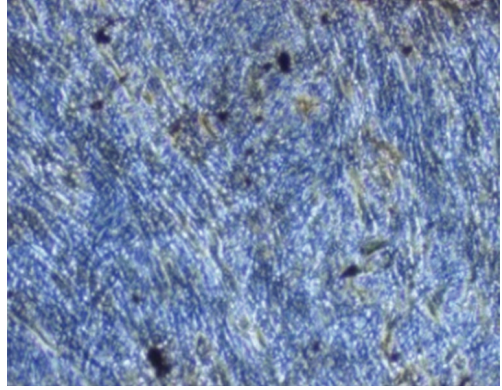

(a)

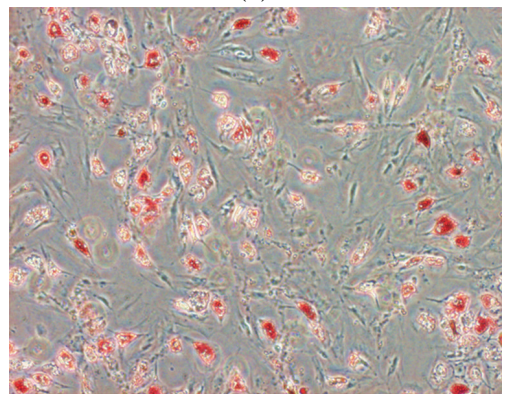

(d)

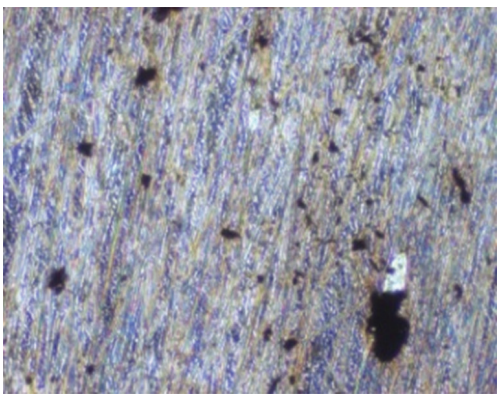

(b)

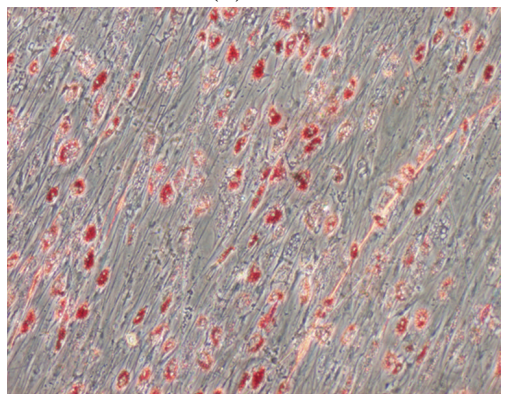

(e)

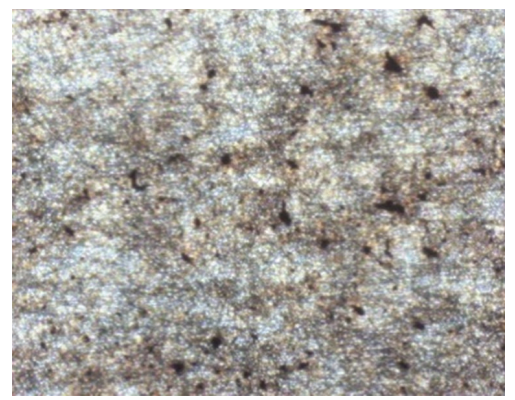

(c)

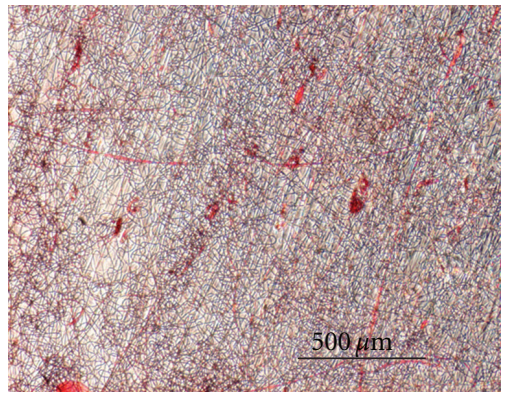

(f)

(A)

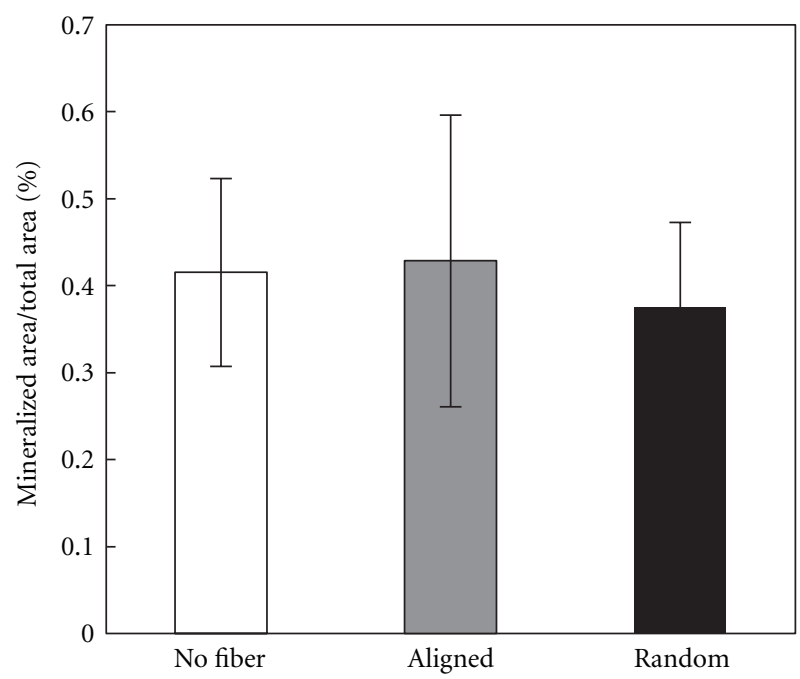

(B)

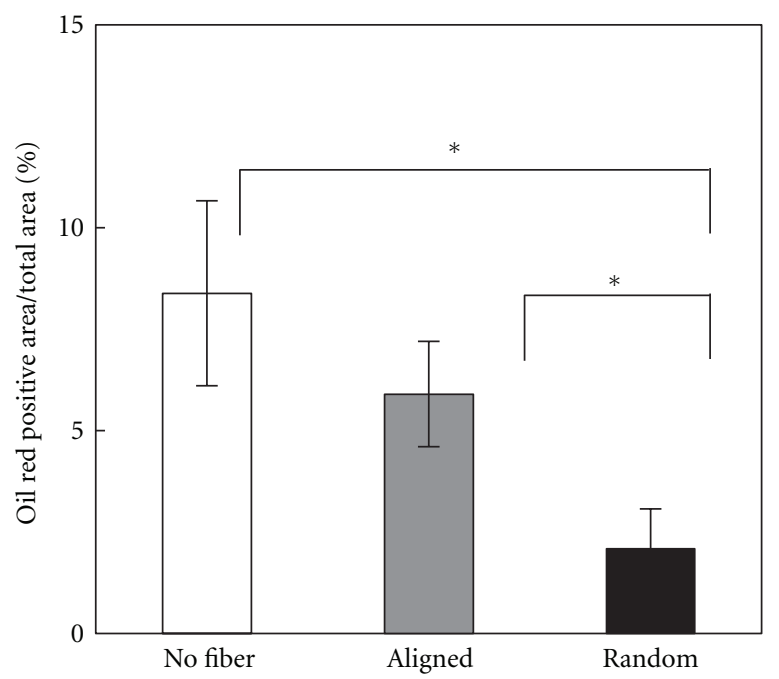

(C)

FIgURE 2: Differentiation of MSCs cultured on nanofibers in OIM or AIM for 14 days. (A) Phase contrast images of differentiated MSCs. ((a)-(c)) MSCs differentiated with OIM, stained by von Kossa method and ((d)-(f)) MSC differentiated with AIM, stained by Oil-Red-O. Cells were cultured on a flat dish ((a) and (d)), aligned fibers ((b) and (e)), and random fibers. ((c) and (f)) Bar $=500 \mu \mathrm{m}$. (B) Quantitative analysis of osteoinduction. (C) Quantitative analysis of adipoinduction. (*: $P<0.05)$.

calcium deposition between the 2 types of scaffolds. However, after adipoinduction using AIM, cells on random fibers appeared to be less differentiated than cells grown on aligned nanofibers, despite having been cultured in the presence of the same soluble adipogenic factors (Figures 2(A) and 2(C)).

To examine the differentiation of MSCs at a genetic level, RT-PCR was also performed. Osteoinduced MSCs cultured on the different types of nanofibers showed no significant difference in terms of the expression of osteogenic markers from cells that had been cultured on a flat dish, but adipoinduced MSCs cultured on these various substrates showed different expression levels of adipogenic markers (Figure 3). Specifically, the expression of adiponectin (ADIPOQ), [19, 20] a hormone secreted specifically from adipose tissue, and lipoprotein lipase (LPL), 1 of the 5 major groups of lipoproteins, [21] were inhibited in cells grown on nanofiber scaffolds compared to those grown on the flat dish. Moreover, peroxisome proliferator-activated receptor gamma 


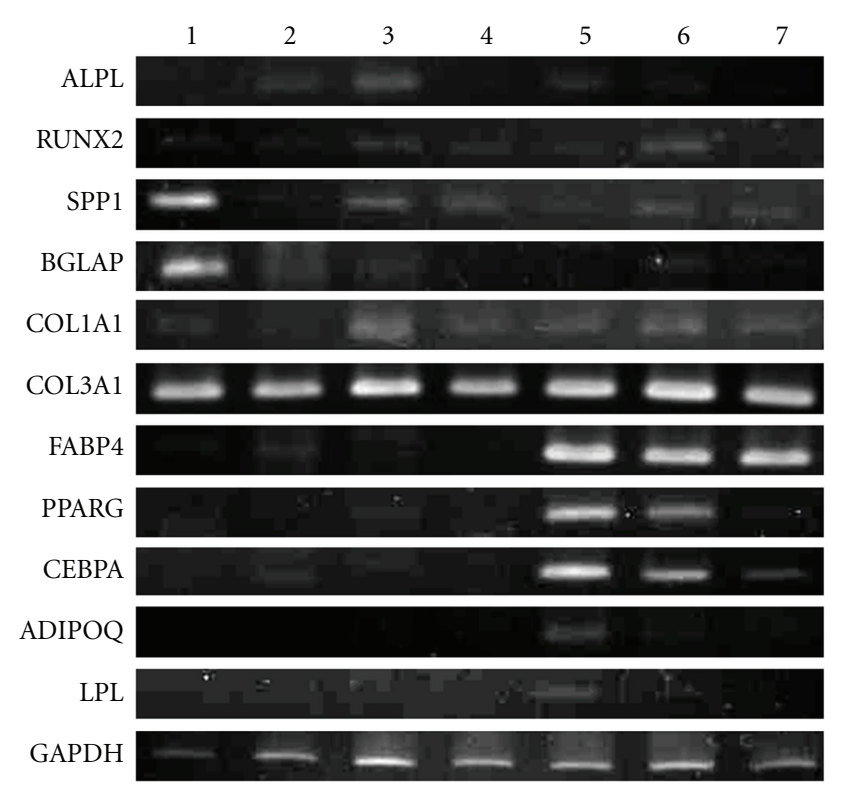

FIGURE 3: Gene expression of the differentiated MSCs at day 3. Lane 1: MSC cultured in DMEM; lanes 2-4: MSCs cultured in OIM; lanes 5-7: MSC cultured in AIM. Cells were cultured in a dish (lanes 1, 2, and 5), aligned nanofibers (lanes 3 and 6), random nanofibers. (lanes 4 and 7) ALPL: alkaline phosphatase; RUNX2: runt-related transcription factor 2; SPP1: secreted phosphoprotein 1 (osteopontin); BGLAP: bone gamma-carboxyglutamate (gla) protein; COL1A1: collagen, type I, alpha 1; COL3A1: collagen, type III, alpha 1; FABP4: fatty acid binding protein 4; PPARG: peroxisome proliferator-activated receptor gamma; CEBPA: CEBPA CCAAT/enhancer binding protein (C/EBP), alpha; ADIPOQ: adiponectin, C1Q, and collagen domain containing; LPL: lipoprotein lipase; GAPDH: glyceraldehyde-3-phosphate dehydrogenase (as standard).

(PPARG), which regulates fatty acid storage and glucose metabolism, $[19,20]$ and CCAAT/enhancer-binding protein alpha (CEBPA), which transactivates the promotors of several adipocyte markers, [22] were inhibited more strongly on a random than on an aligned scaffold, which was consistent with histochemical result mentioned above.

3.3. Geometric Control of the Differentiation of MSCs. To investigate whether MSC differentiation could be controlled by the geometry of the scaffold, we induced differentiation using a mixture of OIM and AIM, which can induce cells into both adipocytes and osteoblasts. As in the previous experiment, we placed the 2 types of scaffolds into the same dish, in order that the cells on either scaffold would share the same medium. As a result of the mixed medium, the MSCs cultured on a flat dish showed differentiation into both adipocytes and osteoblasts (Figure 4). On the other hand, MSCs cultured on the different nanofiber scaffolds showed less differentiation into adipocytes but showed no difference in terms of the percentage of cells that differentiated into osteoblasts on this scaffold as compared to the percentage of osteoblasts produced on the flat dish surface in the mixed medium. More particularly, MSCs grown on random nanofibers showed mainly osteoinduction, and markedly less adipoinduction, even though the medium also contained soluble factors that promote adipoinduction. This indicated that direct control of the commitment of MSCs to differentiate into a particular cell type had been achieved through the geometry of the scaffold.
3.4. DNA Microarray Analysis. To evaluate the effect of scaffold geometry on MSC differentiation at a genetic level, we compared gene expression in MSCs that had been cultured on the aligned and random nanofiber scaffolds in maintaining medium for 3 days, using a DNA microarray that can assess the expression of 28,869 genes. The result of the DNA microarray is summarized in Figure 5. In this figure, we listed the genes of which expression levels were 1.5-fold different between the MSCs cultured on the aligned and random nanofibers. Specifically, we identified 27 genes that showed a higher expression in MSCs cultured on aligned fibers, and 20 genes that showed higher expression in MSCs cultured on random fibers.

In particular, we found differential expression of secretable soluble proteins through the geometry of the scaffold. One of these proteins was interleukin 21 (IL-21), a cytokine which plays a role in the proliferation and maturation of natural killer (NK) cells. [23] Serpin peptidase inhibitor, which helps to control cell function by blocking the activity of peptidases, $[24,25]$ was also enhanced in cells grown on aligned nanofibers. On the other hand, keratinocyte growth factor-like protein, a type of heparin-binding growth factor previously known as FGF-7, [26] was enhanced in cells grown on random fibers. This protein is reported to play a role in differentiation of epidermal stem/progenitors into keratinocytes, as well as in DNA synthesis, and cell proliferation. [27-29] Thus, MSC differentiation may be affected by the balance of these soluble factors.

We also found differential expression of transcriptional factors. The expression of POU class 5 homeobox 1B, also 


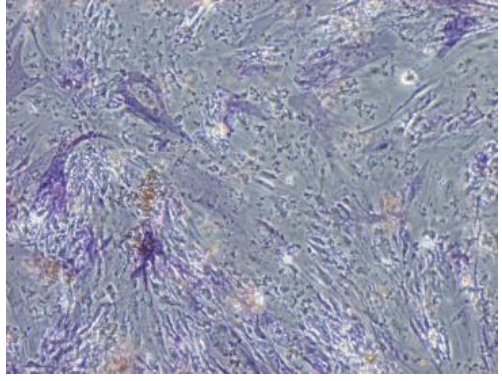

(a)

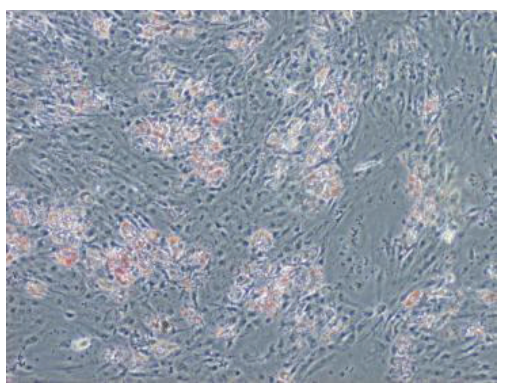

(d)

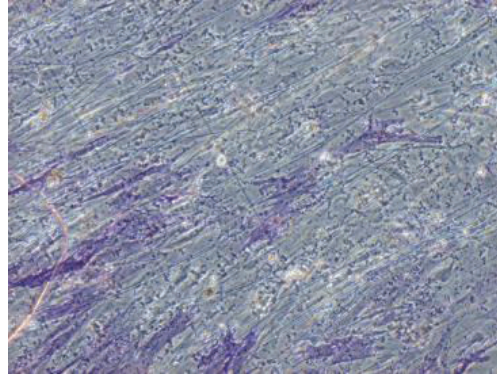

(b)

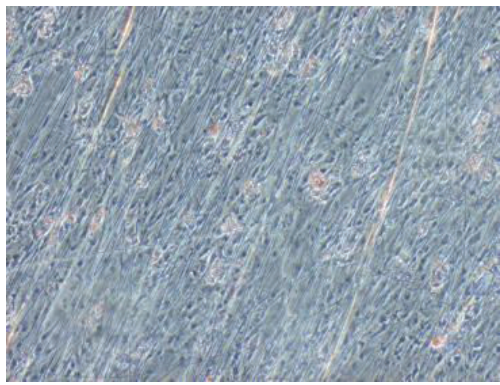

(e)

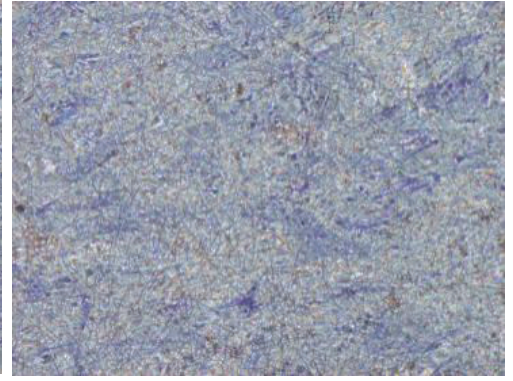

(c)

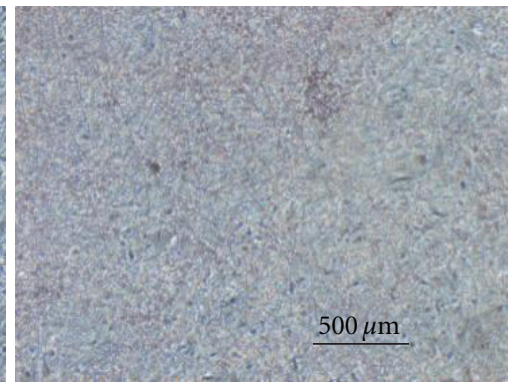

(f)

(A)

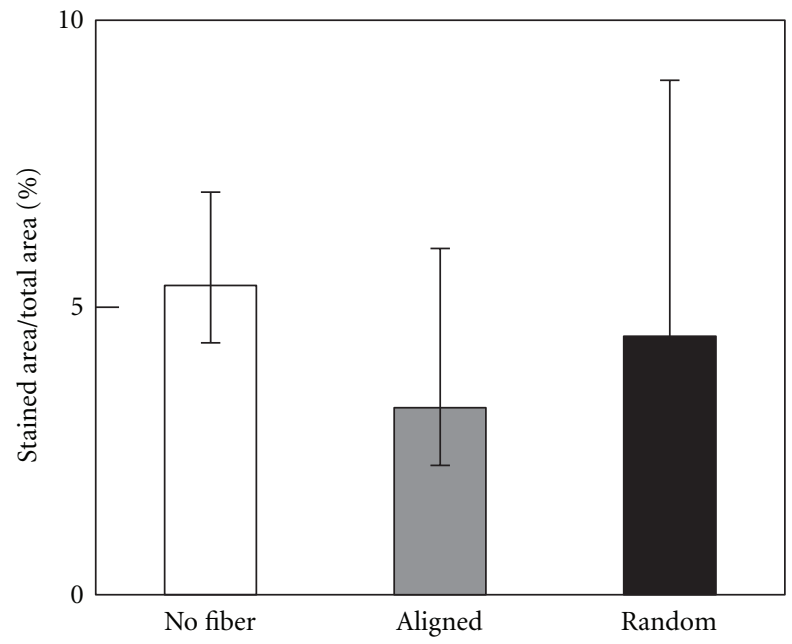

(B)

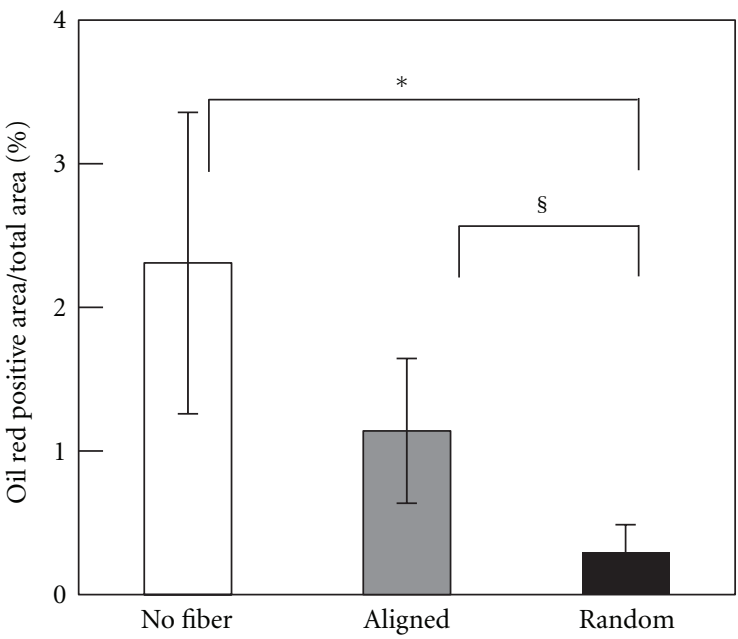

(C)

FIGURE 4: Differentiation of MSC on nanofiber in the mixed medium for 7 days. (A) Phase contrast images of differentiated MSC. MSCs were stained by alkaline phosphatase ((a)-(c)) and with Oil-Red-O. ((d)-(f)) Cells were cultured on a flat dish, ((a) and (d)) aligned fibers, ((b) and (e)) and random fibers. ((c) and (f)) Bar $=500 \mu \mathrm{m}$. (B) Quantitative analysis of osteoinduction. (C) Quantitative analysis of adipoinduction. $\left({ }^{*}: P<0.05 ;{ }^{\S}: P<0.1\right)$.

known as Oct-3/4, which is a key transcriptional factor in the maintenance of stem cell [30, 31], was enhanced on aligned fibers. We also found that the expressions of some subfamilies of zinc-finger proteins were altered; specifically, those with the C2H2 DNA-binding motif characteristic of transcriptional factors, which are functionally important regulators of development [32]. It is possible that changes in these transcriptional factors trigger an intracellular cascade resulting in differentiation. However, we did not find any significant changes in cytoskeletal factors, as had previously been reported.

\section{Conclusion}

In this study, we demonstrated that differentiation of MSCs is affected by the geometry of a nanofiber scaffold. Induction of differentiation of MSCs into adipocytes was markedly more inhibited on random than on aligned nanofibers. We also showed that specific control of differentiation is possible through manipulating geometry in the presence of a mixed medium that facilitates both adipoinduction and osteoinduction. Although osteoinduction in the mixed medium was almost equal between the geometrically different scaffolds, 


\begin{tabular}{|c|c|c|c|c|c|c|}
\hline $\begin{array}{l}\text { Expression in } \\
\text { aligned MSC }\end{array}$ & $\begin{array}{l}\text { Expression in } \\
\text { random MSC }\end{array}$ & $\begin{array}{c}\text { Fold } \\
\text { change }\end{array}$ & Gene name & Gene family & $\begin{array}{l}\text { Approved } \\
\text { symbol }\end{array}$ & HGNC ID \\
\hline & & 0.6641 & Defensin, $\beta 113$ & Defensins, $\beta$ & DEFB113 & HGNC:18094 \\
\hline & & 0.6405 & Olfactory receptor, family 6 , subfamily $\mathrm{C}$, member 2 & Olfactory receptors & OR6C2 & HGNC:15436 \\
\hline & & 0.4821 & Heat shock protein $90 \mathrm{kDa} \alpha$ (cytosolic), class A member 6 , pseudogene & - & HSP90AA6P & HGNC:32536 \\
\hline & & 0.6275 & Adenylate cyclase 10 (soluble) pseudogene & - & ADCY10 & HGNC:21285 \\
\hline & & 0.6504 & Interleukin 21 & Interleukins and interleukin receptors & IL21 & HGNC:6005 \\
\hline & & 0.6289 & Cytochrome P450, family 2, subfamily B, polypeptide 7 pseudogene 1 & Cytochrome P450s & CYP2B7P1 & HGNC:2616 \\
\hline & & 0.6217 & Enkurin, TRPC channel interacting protein & - & ENKUR & HGNC:28388 \\
\hline & & 0.6658 & Zinc finger protein 594 & Zinc fingers, $\mathrm{C} 2 \mathrm{H} 2$-type & ZNF594 & HGNC:29392 \\
\hline & & 0.6544 & Hydroxy- $\delta$-5-steroid dehydrogenase, $3 \beta$-and steroid $\delta$-isomerase 2 & Short chain dehydrogenase/reductase superfamily/extended SDR fold & HSD3B2 & HGNC:5218 \\
\hline & & 0.66 & Small nuclear ribonucleoprotein $40 \mathrm{kDa}$ (U5) & WD repeat domain containing & SNRNP40 & HGNC:3085 \\
\hline & & 0.5982 & Olfactory receptor, family 1 , subfamily Q, member 1 & Olfactory receptors & ORIQ1 & HGNC:8223 \\
\hline & & 0.5497 & \begin{tabular}{|l|} 
Thioredoxin domain containing 9 \\
\end{tabular} & - & TXNDC9 & HGNC:24110 \\
\hline & & 0.6304 & Target of mybl (chicken)-like 1 & & & \\
\hline & & 0.6499 & Family with sequence similarity 185 , member A & - & FAM185A & HGNC:22412 \\
\hline & & 0.6574 & RNA, U5B small nuclear 1 & ncRNAs/small nuclear and cytoplasmic RNAs: Nuclear & RNU5B-1 & \\
\hline & & 0.5933 & Long intergenic non-protein coding RNA 518 & ncRNAs/long non-coding RNAs & LINC00518 & HGNC:28626 \\
\hline & & 0.6207 & \begin{tabular}{|l|} 
Coiled-coil domain containing 59 \\
\end{tabular} & - & CCDC59 & HGNC:25005 \\
\hline & & 0.6418 & SPRY domain containing 5 & - & SPRYD5 & HGNC:19023 \\
\hline & & 0.6599 & Cytochrome P450, family 4, subfamily A, polypeptide 11 & Cytochrome P450s & CYP4A11 & HGNC:2642 \\
\hline & & 0.6535 & High-mobility group box 1 & High-mobility group/canonical & HMGB1 & HGNC:4983 \\
\hline & & 0.6329 & Golgin A6 family, member A & - & GOLGA6A & HGNC:13567 \\
\hline & & 0.6449 & Eukaryotic translation elongation factor $1 \alpha 1$ & - & EEFIAl & HGNC:3189 \\
\hline & & 0.6127 & Hypothetical LOC440944 & & & \\
\hline & & 0.644 & Sialic acid binding Ig-like lectin 16 (gene/pseudogene) & Sialic acid binding Ig-like lectins & SIGLEC16 & HGNC:24851 \\
\hline & & 0.6549 & POU class 5 homeobox $1 \mathrm{~B}$ & Homeoboxes/POU class & POU5F1B & HGNC:9223 \\
\hline & & 0.6441 & Small nucleolar RNA, C/D box 6 & ncRNAs/small nucleolar RNAs: C/D box containing & SNORD6 & HGNC:32703 \\
\hline & & 0.6062 & Zinc finger protein 525 & Zinc fingers, C2H2-type & ZNF525 & HGNC:29423 \\
\hline & & 1.545 & Microfibrillar associated protein 5 & - & MFAP5 & HGNC:29673 \\
\hline & & 1.507 & Transmembrane $4 \mathrm{~L}$ six family member 1 & - & TM4SF1 & HGNC:11853 \\
\hline & & 1.509 & Solute carrier family 14 (urea transporter), member 1 (Kidd blood group) & Blood group antigens; solute carriers & SLC14AI & HGNC:10918 \\
\hline & & 1.722 & Hypothetical LOC100128252 & & & \\
\hline & & 1.56 & Ribosomal protein L41 & L ribosomal proteins & RPL41 & HGNC:10354 \\
\hline & & 1.525 & Serpin peptidase inhibitor, clade B (ovalbumin), member 2 & Serine (or cysteine) peptidase inhibitors & SERPINB2 & HGNC:8584 \\
\hline & & 1.503 & Translocase of inner mitochondrial membrane domain containing 1 & - & TIMMDC1 & HGNC:1321 \\
\hline & & 1.641 & RAB guanine nucleotide exchange factor (GEF) 1 & - & RABGEF1 & HGNC:17676 \\
\hline & & 1.515 & Tumor necrosis factor, $\alpha$ induced protein 6 & - & TNFAIP6 & HGNC:11898 \\
\hline & & 1.658 & WD repeat domain 33 & & & \\
\hline & & 1.548 & Protease, serine, 1 (trypsin 1) & Serine peptidases/serine peptidases & PRSSI & HGNC:9475 \\
\hline & & 1.502 & Hypothetical LOC400590 & & & \\
\hline & & 1.555 & Pleiomorphic adenoma gene-like 2 & Zinc fingers, $\mathrm{C} 2 \mathrm{H} 2$-type & PLAGL2 & HGNC:9047 \\
\hline & & 1.869 & Zinc finger protein 253 & Zinc fingers, $\mathrm{C} 2 \mathrm{H} 2$-type & ZNF253 & HGNC:13497 \\
\hline & & 1.697 & Fanconi anemia, complementation group $\mathrm{M}$ & Fanconi anemia, complementation groups & FANCM & HGNC:23168 \\
\hline & & 1.635 & Solute carrier family 25 , member 40 & Solute carriers & SLC25A40 & HGNC:29680 \\
\hline & & 1.699 & Zinc finger protein 805 & Zinc fingers, $\mathrm{C} 2 \mathrm{H} 2$-type & ZNF805 & HGNC:23272 \\
\hline & & 1.684 & Olfactory receptor, family 4, subfamily C, member 45 & Olfactory receptors & OR4C45 & HGNC:3127 \\
\hline & & 1.576 & Keratinocyte growth factor-like protein 1 & Heparin-binding growth factors & KGFLP1 & \\
\hline & & 2.825 & RNA, U5A small nuclear & ncRNAs / small nuclear and cytoplasmic RNAs: nuclear & RNU5A-1 & HGNC:10211 \\
\hline
\end{tabular}

FiguRE 5: Results of DNA microarray. Gene expression levels were shown in a heat map. Genes listed in a upper part were expressed higher in aligned MSC, while genes listed in a lower part were expressed higher in random MSCs.

adipoinduction was markedly inhibited on random nanofibers. We further examined gene expression in these cells by DNA microarray and found that several types of transcriptional factors and soluble factors were affected by the geometry of the nanofiber scaffold. Thus, commitment of MSCs to differentiation into a particular cell lineage can be controlled by scaffold geometry, and not only by using particular soluble factors in the medium. This approach is expected to be useful as it may allow precise and localized control of MSC differentiation by manufactured materials used in tissue engineering.

\section{Acknowledgments}

A part of this work was supported by the JGC-S Scholarship Foundation, no. 2319. It was further supported by Grant-inAid for Research Activity Start-up, MEXT, no. 23850009. The authors thank Dr. Kazuhiro Karaya (Life Science Research Laboratory, University of Fukui) for DNA microarray analysis.

\section{References}

[1] M. F. Pittenger, A. M. Mackay, S. C. Beck et al., "Multilineage potential of adult human mesenchymal stem cells," Science, vol. 284, no. 5411, pp. 143-147, 1999.

[2] F. P. Barry and J. M. Murphy, "Mesenchymal stem cells: clinical applications and biological characterization," International Journal of Biochemistry and Cell Biology, vol. 36, no. 4, pp. 568584, 2004.

[3] C. D. Porada, E. D. Zanjani, and G. Almeida-Porad, "Adult mesenchymal stem cells: a pluripotent population with multiple applications," Current Stem Cell Research \& Therapy, vol. 1, no. 3, pp. 365-369, 2006.

[4] K. Le Blanc and O. Ringdén, "Immunomodulation by mesenchymal stem cells and clinical experience," Journal of Internal Medicine, vol. 262, no. 5, pp. 509-525, 2007.

[5] S. E. Hanson, K. A. Gutowski, and P. Hematti, "Clinical applications of mesenchymal stem cells in soft tissue augmentation," Aesthetic Surgery Journal, vol. 30, no. 6, pp. 838-842, 2010.

[6] A. Sterodimas, J. De Faria, W. E. Correa, and I. Pitanguy, "Tissue engineering in plastic surgery: an up-to-date review 
of the current literature," Annals of Plastic Surgery, vol. 62, no. 1, pp. 97-103, 2009.

[7] F. M. Chen, J. Zhang, M. Zhang, Y. An, F. Chen, and Z. F. Wu, "A review on endogenous regenerative technology in periodontal regenerative medicine," Biomaterials, vol. 31, no. 31, pp. 7892-7927, 2010.

[8] R. McBeath, D. M. Pirone, C. M. Nelson, K. Bhadriraju, and C. $\mathrm{S}$. Chen, "Cell shape, cytoskeletal tension, and RhoA regulate stem cell lineage commitment," Developmental Cell, vol. 6, no. 4, pp. 483-495, 2004.

[9] D. S. W. Benoit, M. P. Schwartz, A. R. Durney, and K. S. Anseth, "Small functional groups for controlled differentiation of hydrogel-encapsulated human mesenchymal stem cells," Nature Materials, vol. 7, no. 10, pp. 816-823, 2008.

[10] N. Fujimoto, S. Fujita, T. Tsuji et al., "Microencapsulated feeder cells as a source of soluble factors for expansion of CD $34^{+}$hematopoietic stem cells," Biomaterials, vol. 28, no. 32, pp. 4795-4805, 2007.

[11] Q. P. Pham, U. Sharma, and A. G. Mikos, "Electrospinning of polymeric nanofibers for tissue engineering applications: a review," Tissue Engineering, vol. 12, no. 5, pp. 1197-1211, 2006.

[12] A. Greiner and J. H. Wendorff, "Electrospinning: a fascinating method for the preparation of ultrathin fibers," Angewandte Chemie-International Edition, vol. 46, no. 30, pp. 5670-5703, 2007.

[13] T. J. Sill and H. A. von Recum, "Electrospinning: applications in drug delivery and tissue engineering," Biomaterials, vol. 29, no. 13, pp. 1989-2006, 2008.

[14] L. Marinucci, S. Balloni, E. Becchetti et al., "Effects of hydroxyapatite and biostite on osteogenic induction of hMSC," Annals of Biomedical Engineering, vol. 38, no. 3, pp. 640-648, 2010.

[15] S. Fujita, J. Toguchida, Y. Morita, and H. Iwata, "Clonal analysis of hematopoiesis-supporting activity of human mesenchymal stem cells in association with Jagged 1 expression and osteogenic potential," Cell Transplantation, vol. 17, no. 1011, pp. 1169-1179, 2008.

[16] H. N. Yang, J. S. Park, D. G. Woo et al., "C/EBP- $\alpha$ and C/EBP- $\beta$-mediated adipogenesis of human mesenchymal stem cells (hMSCs) using PLGA nanoparticles complexed with poly (ethyleneimmine)," Biomaterials, vol. 32, no. 25, pp. 59245933, 2011.

[17] C. J. Heffelfinger and R. L. Burton, "X-Ray determination of the crystallite orientation distributions of polyethylene terephthalate films," Journal of Polymer Science, vol. 47, no. 149, pp. 289-306, 1960.

[18] S. Fujita, D. Ono, M. Ohshima, and H. Iwata, "Supercritical $\mathrm{CO}_{2}$-assisted embossing for studying cell behaviour on microtextured surfaces," Biomaterials, vol. 29, no. 34, pp. 4494-4500, 2008.

[19] M. Stumvoll and H. Häring, "The peroxisome proliferatoractivated receptor- $\gamma 2$ Pro12Ala polymorphism," Diabetes, vol. 51, no. 8, pp. 2341-2347, 2002.

[20] P. Ferré, "The biology of peroxisome proliferator-activated receptors: relationship with lipid metabolism and insulin sensitivity," Diabetes, vol. 53, supplement 1, pp. S43-S50, 2004.

[21] G. J. Hausman, D. R. Campion, and R. J. Martin, "Search for the adipocyte precursor cell and factors that promote its differentiation," Journal of Lipid Research, vol. 21, no. 6, pp. 657670, 1980.

[22] M. D. Lane, F. T. Lin, O. A. MacDougald, and M. VasseurCognet, "Control of adipocyte differentiation by CCAAT/ enhancer binding protein $\alpha(\mathrm{C} / \mathrm{EBP} \alpha)$," International Journal of Obesity, vol. 20, supplement 3, pp. S91-S96, 1996.
[23] J. Parrish-Novak, S. R. Dillon, A. Nelson et al., "Interleukin 21 and its receptor are involved in NK cell expansion and regulation of lymphocyte function," Nature, vol. 408, no. 6808, pp. 57-63, 2000.

[24] N. El Haddad, D. Heathcote, R. Moore et al., "Mesenchymal stem cells express serine protease inhibitor to evade the host immune response," Blood, vol. 117, no. 4, pp. 1176-1183, 2011.

[25] C. Chiellini, O. Cochet, L. Negroni et al., "Characterization of human mesenchymal stem cell secretome at early steps of adipocyte and osteoblast differentiation," BMC Molecular Biology, vol. 9, article 26, 2008.

[26] H. Steiling and S. Werner, "Fibroblast growth factors: key players in epithelial morphogenesis, repair and cytoprotection," Current Opinion in Biotechnology, vol. 14, no. 5, pp. 533-537, 2003.

[27] J. S. Rubin, H. Osada, P. W. Finch, W. G. Taylor, S. Rudikoff, and S. A. Aaronson, "Purification and characterization of a newly identified growth factor specific for epithelial cells," Proceedings of the National Academy of Sciences of the United States of America, vol. 86, no. 3, pp. 802-806, 1989.

[28] R. J. Panos, J. S. Rubin, S. A. Aaronson, and R. J. Mason, "Keratinocyte growth factor and hepatocyte growth factor/scatter factor are heparin-binding growth factors for alveolar type II cells in fibroblast-conditioned medium," Journal of Clinical Investigation, vol. 92, no. 2, pp. 969-977, 1993.

[29] S. Aguilar, C. J. Scotton, K. McNulty et al., "Bone marrow stem cells expressing keratinocyte growth factor via an inducible lentivirus protects against bleomycin-induced pulmonary fibrosis," PLoS ONE, vol. 4, no. 11, Article ID e8013, 2009.

[30] M. Pesce and H. R. Schöler, "Oct-4: gatekeeper in the beginnings of mammalian development," Stem Cells, vol. 19, no. 4, pp. 271-278, 2001.

[31] M. Abu-Remaileh, A. Gerson, M. Farago et al., "Oct-3/4 regulates stem cell identity and cell fate decisions by modulating Wnt/ $\beta$-catenin signalling," The EMBO Journal, vol. 29, no. 19, pp. 3236-3248, 2010.

[32] B. Ganss and A. Jheon, "Zinc finger transcription factors in skeletal development," Critical Reviews in Oral Biology and Medicine, vol. 15, no. 5, pp. 282-297, 2004. 

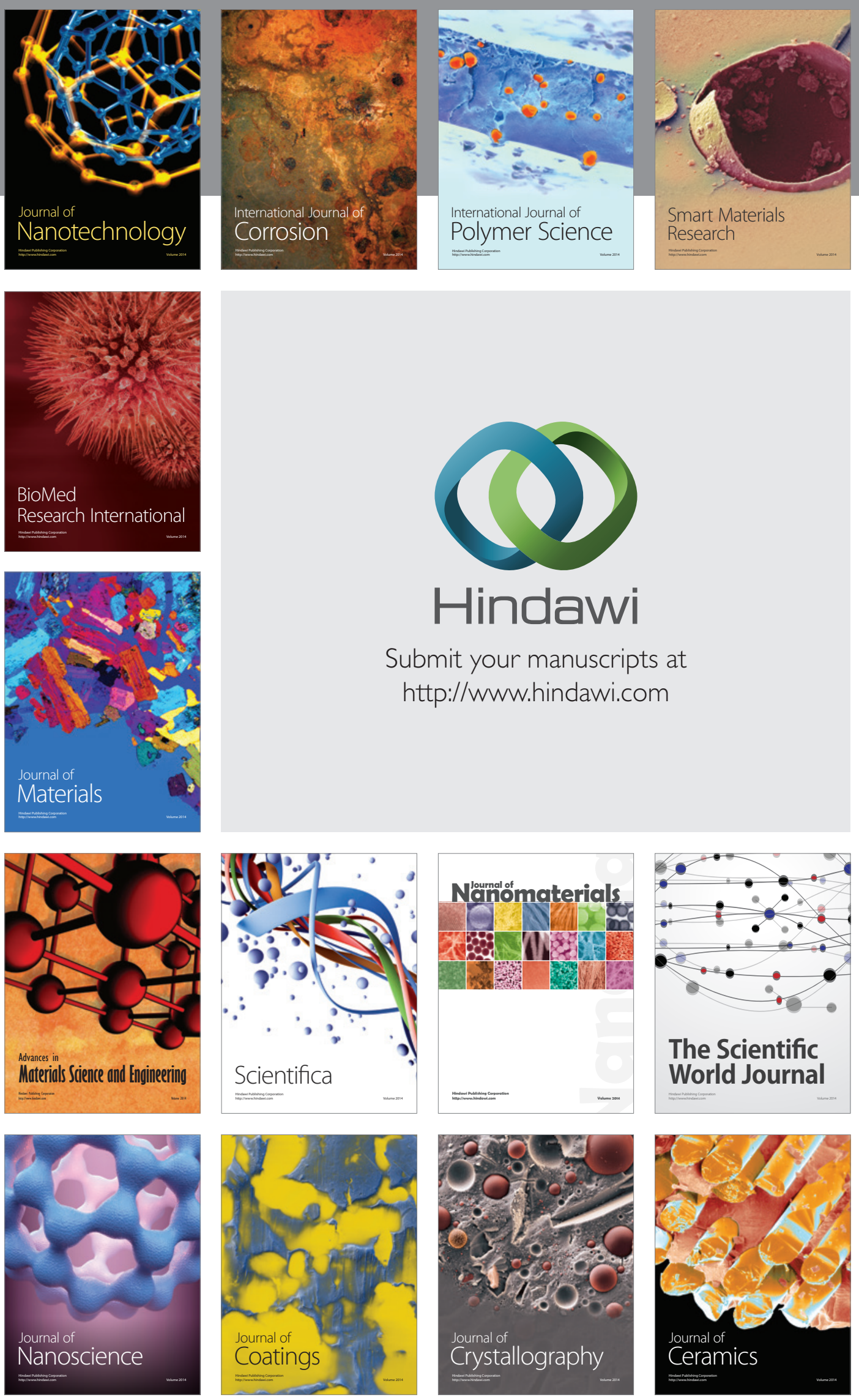

The Scientific World Journal

Submit your manuscripts at

http://www.hindawi.com

\section{World Journal}

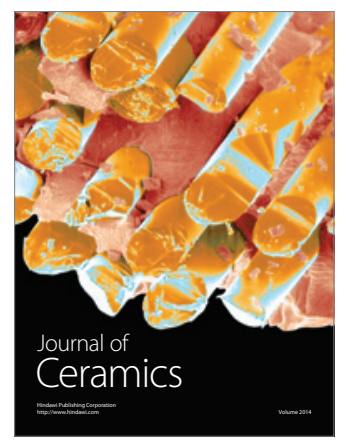

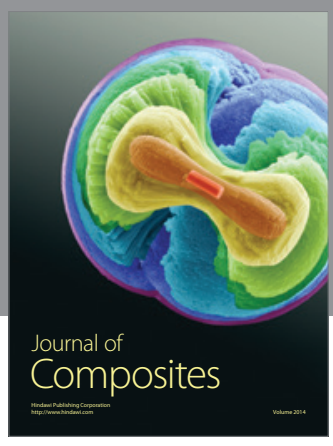
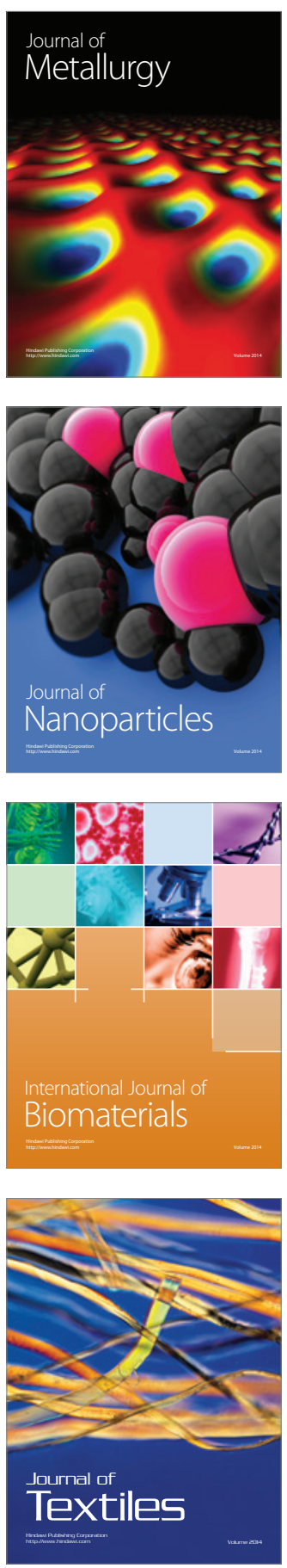